\title{
PATHOLOGY
}

\section{Clinical benefit of scrotal specimen analysis questioned}

An investigation into the rate of malignancy detected in hydrocele and spermatocele specimens suggests that routine pathological analysis of these surgical samples is not clinically beneficial, and incurs considerable cost to the patient or health-care system.

Viral Shah and colleagues at the University of Iowa reviewed the medical records of 264 patients who underwent surgery for hydrocele or spermatocele at their institution between January 2000 and August 2013. They found that a large proportion of samples were sent for pathological analysis: $51 \%$ and $90 \%$ of all hydrocelectomy and spermatocelectomy specimens, respectively. However, none of the excised tissues harboured any abnormalities according to the pathology report (atypia, dysplasia, increase in mitoses, carcinoma, tumour or malignancy), leading the researchers to question the clinical value of this practice.

Furthermore, cost analysis-based on the most common Current Procedural
Terminology code for pathological analysis-revealed an estimated total cost of US $\$ 49,449$ for this cohort over the 14-year period ( $\$ 26,418$ for hydrocelectomies and $\$ 17,727$ for spermatocelectomies). A cost:benefit ratio could not be calculated owing to the absence of any malignant specimens.

Although these data might not reflect current practice at other institutions, they should encourage surgeons to question whether pathological analysis is required for scrotal specimens from all patients. Shah et al. suggest reserving such analyses for suspicious cases, including men who have a history of asbestos exposure or evidence of hydrocele plus papillary projections on ultrasonography.

Sarah Payton

Original article Shah, V. S. et al. Routine pathologic evaluation of hydrocele and spermatocele specimens is associated with significant costs and no identifiable benefit. J. Urol. doi:10.1016/j.juro.2014.04.085 